\title{
La inmunología en Antioquia: una historia con futuro. III. El presente y los retos del futuro
}

\author{
Luis F. García
}

\section{RESUMEN}

La inmunología es una de las ciencias biomédicas con mayor desarrollo en la segunda parte del siglo XX y principios del presente en al ámbito mundial. También ha tenido un desarrollo muy importante en Antioquia a partir de los años sesenta del siglo pasado. En la tercera parte de esta reseña histórica el autor expone, según su criterio, cuál es el estado actual y los retos futuros de la inmunología en lo científico, pedagógico y administrativo en la Universidad de Antioquia.

\section{PALABRAS CLAVE}

Antioquia; Historia; Inmunología

\section{SUMMARY}

\begin{abstract}
Immunology in Antioquia: a history with future. III. It's present and challenges for the future

Immunology is one of the biomedical sciences that had, worldwide, a greater development in the last part of the XX century and the beginning of the present century. In Antioquia, immunology also had important developments that began in the 1960s. In the third part of this historical review, the author exposes his personal view about the present situation and the future challenges of immunology at the scientific, pedagogical and administrative levels in the Universidad de Antioquia.
\end{abstract}

1 Profesor emérito, Grupo de Inmunología Celular e Inmunogenética, Facultad de Medicina, Sede de Investigación Universitaria, Universidad de Antioquia, Medellín, Colombia. Correspondencia: Luis F. García; Ifgarcia@une.net.co

Recibido: abril 12 Aceptado: julio18

Cómo citar: García LF. La inmunología en Antioquia: una historia con futuro. III. El presente y los retos del futuro. latreia. 2020 Jul-Sep;33(3):298-304. D0I 10.17533/udea.iatreia.43. 


\section{KEYWORDS}

Antioquia; History; Immunology

\section{INTRODUCCIÓN}

En la primera y segunda parte de esta reseña histórica de la inmunología en Antioquia relaté los hechos ocurridos entre 1963 y mediados de los años noventa que llevaron a que la inmunología sea hoy una de las disciplinas biomédicas más desarrolladas en el ámbito científico local. En esta última sección haré una breve descripción del estado actual, los mayores logros y los principales reconocimientos recibidos por los grupos y los investigadores en esta área, plantearé algunas consideraciones críticas, desde mi punto de vista, sobre las actividades investigativas y docentes que estos grupos realizan y finalmente, expondré algunos retos que considero pueden ser importantes para continuar y consolidar, aún más la inmunología que se hace en Antioquia en el contexto nacional e internacional.

\section{El estado actual de la inmunología en la Universi- dad de Antioquia}

A partir de mediados de los años noventa, con la conformación de los grupos de investigación, la creación del posgrado en Ciencias Básicas Biomédicas (transformado luego en Corporación Académica) con sus nuevos programas de maestría y doctorado, la adquisición del primer citómetro de flujo y la incorporación de nuevos docentes con formación en investigación, ya fuera porque regresaban al país en el Programa de Retorno financiado por Colciencias o por el compromiso institucional de vincular a quienes llevaron a cabo su doctorado con becas de esta entidad, se dio un salto cuantitativo y cualitativo muy importante que se reflejó en un claro liderazogo de la inmunología en la investigación de la Universidad. De estas vinculaciones, en ambas modalidades, resalto los nombres de algunos investigadores en inmunología que han impactado positivamente su desarrollo: Gloria Vásquez, Claudia Trujillo, María Teresa Rugeles, Gloria Inés Sánchez, Pablo Javier Patiño, José Luis Franco, Mauricio Rojas, José Robinson Ramírez, CarIos Julio Montoya, María del Pilar Jiménez y Cristiam Álvarez, entre otros. Este liderazó lo demuestran dos hechos históricos: 1. el primer graduado en la Universidad de Antioquia en los programas modernos de doctorado fue Pablo Javier Patiño en 1977, con una tesis dirigida por Diana García de Olarte sobre los mecanismos moleculares y genéticos de la enfermedad granulomatosa crónica y, 2. el otorgamiento, de parte de la Universidad de Antioquia, de la primera distinción summa cum laude a la tesis doctoral de Mauricio Rojas en 1978 sobre la apoptosis de los macrófagos infectados con Mycobacterium tuberculosis, que ture el honor de dirigir.

Merece especial atención la Unidad de Citometría de Flujo, pues se ha convertido en un apoyo tecnológico fundamental para todos los grupos de inmunología, aún para otros que trabajan en disciplinas afines. Como ya mencioné, el primer citómetro de flujo, marca Becton Dickinson, fue adquirido a principios de los años noventa con aportes de la Universidad y de los grupos GICIG, IDP, Inmunovirología y Reproducción que formaron una especie de consorcio, al cual se adhirieron, posteriormente, otros grupos. EI traslado a la Sede de Investigación Universitaria (SIU) y su dirección por Mauricio Rojas, después de retornar de su posdoctorado en la Universidad de Harvard en el 2006, han permitido que la Unidad de Citometría de Flujo sea un modelo de plataforma tecnológica o core lab al servicio de muchos grupos, con metodologías de punta y la asesoría de un investigador experto en citometría con reconocimiento nacional e internacional. Sin embargo, debo dejar mi nota de precaución, porque creo que nos hemos vuelto demasiado "citómetro dependientes", lo cual sionifica que, sin desconocer el valor de estas metodologías, su disponibilidad inmediata ha hecho que quizás no se exploren otras posibilidades valiosas y pertinentes en algunas investigaciones.

En la historia reciente de investigación en la Universidad de Antioquia no es posible desconocer el gran impacto que ha tenido en el desarrollo de la inmunología, y de todas las disciplinas restantes, la construcción y posterior puesta en funcionamiento de la sede de Investigación Universitaria (SIU) en el 2004 (Figura 1). La SIU cuenta con instalaciones modernas y adecuadas para realizar investigación de frontera, también brinda la tranquilidad, que con frecuencia no era posible tener en las otras instalaciones de la Universidad, y la posibilidad de colaborar con 
investigadores distinguidos de otras áreas biomédicas, químicas y físicas, entre otras. Además, otra de estas facilidades de la plataforma tecnológica que ofrece la SIU es el bioterio, con facilidades para albergar animales de laboratorio en condiciones libres de patógenos específicos (SPF) y, también, la disponibilidad de cepas genéticamente definidas o modificadas. EI bioterio es utilizado intensamente por algunos grupos de inmunología que requieren estos animales en sus proyectos de investigación, haciendo mucho más competitiva, internacionalmente, la investigación inmunológica que se realiza en la Universidad de Antioquia. Debo hacer un reconocimiento a José Robinson Ramírez, coordinador del grupo Inmunomodulación y, a la neurobióloga Patricia Cardona por haber dado la lucha ante una burocracia carente de conocimientos técnicos para lograr la disponibilidad del valioso recurso experimental en el bioterio de la SIU.

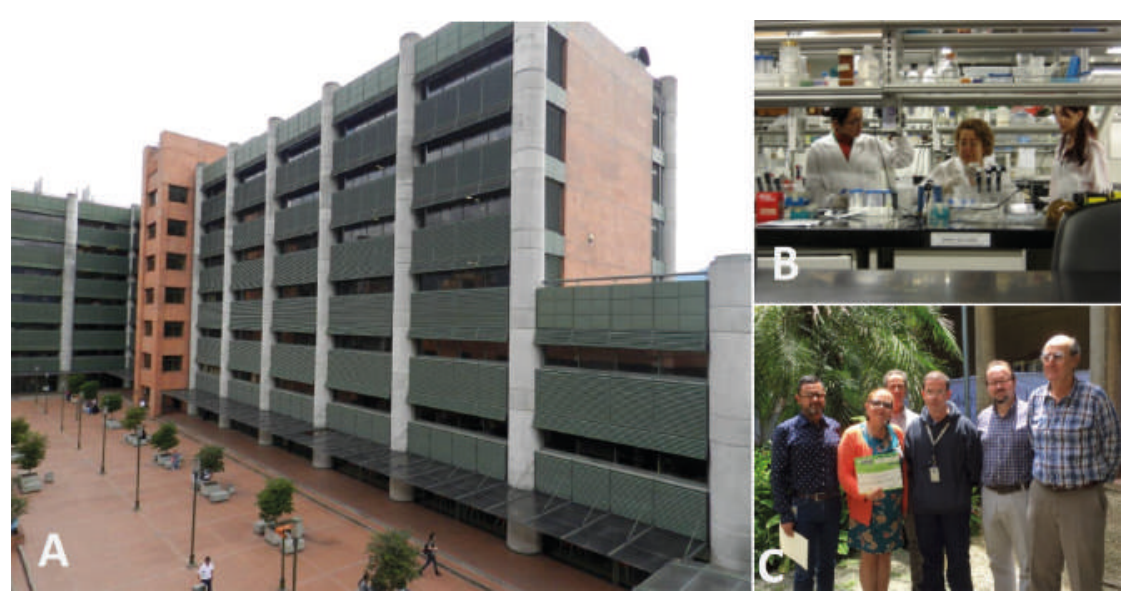

Figura 1. Sede de Investigación Universitaria (SIU) y algunos Profesores-investigadores del Grupo de Inmunología Celular e Inmunogenética (GICIG). A. Edificio de la Sede de Investigación Universitaria (SIU) de la Universidad de Antioquia; B. Sara Claudia París con varios estudiantes en el laboratorio del GICIG en la SIU; C. Profesores-investigadores del GICIG (2018). De izquierda a derecha: Cristiam M. Álvarez, Gloria M. Vásquez, Luis F. Barrera, Mauricio Rojas, Andrés Baena, Luis F. García. Fuente: A, B, Fotografías propias y C, fotografía gentilmente proporcionada por la profesora Gloria Vásquez

Igualmente, Ios proyectos financiados, las publicaciones internacionales en revistas indexadas y las colaboraciones con investigadores de centros de excelencia mundial se incrementaron $y$ permitieron que estos grupos se posicionaran en los niveles más altos del escalafón de Colciencias (Tabla 1), además que, obtuvieran reconocimientos muy importantes dentro de la Universidad (Tabla 2) ${ }^{(1-5)}$, y en el país (Tabla 3). Sin embargo, en el presente ensayo no entraré en los detalles de la historia reciente de estos grupos, pues son ya otros los liderazgos y no creo tener la autoridad para relatar sus respectivas historias. Solo mencionaré tres ejemplos que creo que demuestran el liderazogo de la inmunología que se realiza en la Universidad de Antioquia. El primero es el grupo de Inmunodeficiencias Primarias (IDP), hoy dirigido por José Luis Fran$\mathrm{co}$, que se ha convertido en un centro de referencia
Jeffrey Modell (Jeffrey Modell Foundation for Primary Immunodeficiencies) para estas patologías; el segundo, el Laboratorio de Inmunología de Trasplantes del GICIG, que fue el primero de la Universidad en obtener la acreditación ISO 1725 y, luego, la internacional por la European Federation of Immunogenetics (EFI) para todas las pruebas inmunológicas utilizadas en el estudio de los pacientes que requieren trasplantes de órganos o células hematopoyéticas; $y$, el tercero, el grupo de Alergología Clínica, dirigido por Ricardo Cardona, que creó el primer programa de especialización (residencia) en aleroología clínica del país y, en consecuencia, dio inicio a una escuela de especialistas en esta área de la inmunología con un gran impacto en el manejo de estos trastornos de la respuesta inmune. 
Tabla 1. Grupos de inmunología o con actividades relacionadas con ella de la Universidad de Antioquia. Convocatoria Grupos de Colciencias 2017

\begin{tabular}{|c|c|c|}
\hline Grupo & Categoría & Líneas de investigación \\
\hline $\begin{array}{l}\text { Grupo de Inmunología Celular e Inmuno- } \\
\text { genética (GICIG) }\end{array}$ & A1 & $\begin{array}{l}\text { Respuesta inmune en tuberculosis, inmunología de trasplantes, } \\
\text { autoinmunidad }\end{array}$ \\
\hline $\begin{array}{l}\text { Grupo de Inmunodeficiencias Primarias } \\
\text { (IDP) }\end{array}$ & A1 & Errores innatos de la respuesta inmune \\
\hline Grupo de Inmunovirología & A1 & Inmunología de las infecciones por $\mathrm{VIH}$, dengue y chikungunya \\
\hline Grupo de Reproducción & A & Infertilidad por causas inmunológicas, inmunología de preclamsia \\
\hline Grupo de Infección y Cáncer & A1 & $\begin{array}{l}\text { Respuesta inmune al VPH, inmunología del cáncer de cuello } \\
\text { uterino }\end{array}$ \\
\hline Grupo de Inmunomodulación & B & $\begin{array}{l}\text { Modelos preclínicos de modulación de la respuesta inflamatoria } \\
\text { crónica }\end{array}$ \\
\hline Grupo de Micología Médica & A & Respuesta inmune en micosis humanas \\
\hline Grupo de Alergología Clínica y Experimental & B & Enfermedades alérgicas \\
\hline Grupo de Micología Médica y Experimental & B & $\begin{array}{l}\text { Respuesta inmune en el modelo en el ratón de paracoccidioido- } \\
\text { micosis }\end{array}$ \\
\hline $\begin{array}{l}\text { Centro de Investigaciones Dermatológicas } \\
\text { (CIDERM) }\end{array}$ & $\mathrm{C}$ & Inmunodermatología y neoplasias cutáneas \\
\hline Grupo de Salud y Sociedad & A & Malaria \\
\hline Grupo de Genética Médica & C & Mutaciones en neoplasias del sistema inmune \\
\hline Grupo de Genética Molecular (Genmol) & A1 & Polimorfismos de moléculas del sistema inmune \\
\hline $\begin{array}{l}\text { Programa de Estudio y Control de Enferme- } \\
\text { dades Tropicales (PECET) }\end{array}$ & A1 & Respuesta inmune en Leishmaniasis \\
\hline
\end{tabular}

Fuente: creación propia

Tabla 2. Premios de Investigación de la Universidad de Antioquia concedidos a investigadores o grupos de inmunología

\begin{tabular}{|c|c|c|}
\hline Año & Investigadores galardonados & Producto galardonado \\
\hline 1988 & Luis F. García, Sara C. París & $\begin{array}{l}\text { Taller Latinoamericano de } \\
\text { Histocompatibilidad }\end{array}$ \\
\hline 1998 & $\begin{array}{l}\text { Mauricio Arias, Mauricio Rojas., Jovany Zabaleta, Jaime I. Rodríguez, Sara C. París, } \\
\text { Luis F. Barrera, Luis F. García }\end{array}$ & Ref 1 \\
\hline 2005 & Mauricio Rojas & Ref 2 \\
\hline 2006 & José L. Franco, Pablo J. Patiño, Diana Castaño & Ref 3 \\
\hline 2008 & $\begin{array}{l}\text { Mauricio A. Arias, Gabriela Jaramillo, Yurika P. López, Natalia Mejía, Camila Mejía, } \\
\text { Adelis E. Pantoja, Luis F. García. }\end{array}$ & Ref 4 \\
\hline 2010 & $\begin{array}{l}\text { Helena del Corral, Sara C. París, Nancy D. Marín, Diana M. Marín, Lucelly López, Han- } \\
\text { na M. Henao, Teresita Martínez, Liliana Villa, Luis F. Barrera, Blanca L. Ortiz, María } \\
\text { E. Ramírez, Carlos J. Montes, María C. Oquendo, Lisandra M. Arango, Felipe Riaño, } \\
\text { Carlos Aguirre, Alberto Bustamante, Margarita R, Giraldo, María P. Arbeláez, Carlos } \\
\text { Rojas, Luis F. García }\end{array}$ & Ref. 5 \\
\hline
\end{tabular}

Fuente: Creación propia 
Tabla 3. Algunas distinciones recibidas por investigadores o grupos de inmunología de la Universidad de Antioquia

\begin{tabular}{|c|c|c|c|}
\hline Distinción & Grupo o profesor distinguido & Año & $\begin{array}{l}\text { Institución que } \\
\text { otorga distinción }\end{array}$ \\
\hline Científica del Año en Colombia & Diana García de Olarte & 1986 & $\begin{array}{l}\text { Unión de Ciudadanas de Colombia, } \\
\text { Bogotá }\end{array}$ \\
\hline $\begin{array}{l}\text { Premio Nacional de Medicina "Manuel } \\
\text { Forero" }\end{array}$ & Luis F. García & 1989 & $\begin{array}{l}\text { Academia Nacional de Medicina, Bo- } \\
\text { gotá }\end{array}$ \\
\hline $\begin{array}{l}\text { Premio Alejandro Ángel Escobar en } \\
\text { Ciencias Exactas, Físicas y Naturales }\end{array}$ & $\begin{array}{l}\text { Luis F. García, Mauricio Rojas, } \\
\text { Luis F. Barrera, Jovany Zabale- } \\
\text { ta, Sara C. París, Gloria Vásquez, } \\
\text { Mauricio Arias, Edwin Patiño }\end{array}$ & 2000 & $\begin{array}{l}\text { Fundación Alejandro Ángel Escobar, } \\
\text { Bogotá }\end{array}$ \\
\hline Orden Cámara de Comercio & $\begin{array}{l}\text { Grupo de Inmunología Celular e } \\
\text { Inmunogenética }\end{array}$ & 2000 & $\begin{array}{l}\text { Cámara de Comercio de Medellín, } \\
\text { Medellín }\end{array}$ \\
\hline $\begin{array}{l}\text { Premio Alejandro Ángel Escobar en } \\
\text { Ciencias Exactas, Físicas y Naturales }\end{array}$ & $\begin{array}{l}\text { Pablo J. Patiño y Grupo de Inmu- } \\
\text { nodeficiencias Primarias }\end{array}$ & 2002 & $\begin{array}{l}\text { Fundación Alejandro Ángel Escobar, } \\
\text { Bogotá }\end{array}$ \\
\hline $\begin{array}{l}\text { Premio Anual Alcaldía de Medellín para } \\
\text { el Fomento de la Investigación. Distin- } \\
\text { ción a una vida dedicada a la Investiga- } \\
\text { ción }\end{array}$ & Luis F. García & 2007 & Alcaldía de Medellín, Medellín \\
\hline $\begin{array}{l}\text { Mejor Grupo de Investigación de Edu- } \\
\text { cación Superior }\end{array}$ & $\begin{array}{l}\text { Grupo de Inmunología Celular e } \\
\text { Inmunogenética }\end{array}$ & 2008 & $\begin{array}{l}\text { Ministerio de Educación Nacional, } \\
\text { República de Colombia, Bogotá }\end{array}$ \\
\hline $\begin{array}{l}\text { Premio a la investigación de mayor im- } \\
\text { pacto en el año. Premio Anual Alcaldía } \\
\text { de Medellín para el Fomento de la In- } \\
\text { vestigación }\end{array}$ & $\begin{array}{l}\text { Grupo de Inmunología Celular e } \\
\text { Inmunogenética y Grupo de Epi- } \\
\text { demiología }\end{array}$ & 2009 & Alcaldía de Medellín, Medellín \\
\hline Centro de Referencia Jeffrey Modell & $\begin{array}{l}\text { Grupo de Inmunodeficiencias } \\
\text { Primarias }\end{array}$ & 2010 & $\begin{array}{l}\text { Jeffrey Modell Foundation for Pri- } \\
\text { mary Immunodeficiencies }\end{array}$ \\
\hline $\begin{array}{l}\text { Mejor Investigador Emérito de Colom- } \\
\text { bia. Categoría Ciencias Médicas y de la } \\
\text { Salud }\end{array}$ & Luis F García & 2014 & $\begin{array}{l}\text { Colciencias, Revista Semana, Carta- } \\
\text { gena }\end{array}$ \\
\hline $\begin{array}{l}\text { Premio Anual Alcaldía de Medellín para } \\
\text { el Fomento de la Investigación. Distin- } \\
\text { ción a una vida dedicada a la Investiga- } \\
\text { ción }\end{array}$ & María Teresa Rugeles & 2017 & Alcaldía de Medellín, Medellín \\
\hline $\begin{array}{l}\text { Premio Nacional a la Obra Integral en } \\
\text { Ciencia } 2019\end{array}$ & Luis F García & 2019 & $\begin{array}{l}\text { Academia Colombiana de Ciencias } \\
\text { Exactas Físicas y Naturales y Asocia- } \\
\text { ción Colombiana para el Avance de } \\
\text { la Ciencia, Bogotá }\end{array}$ \\
\hline
\end{tabular}

Fuente: creación propia

\section{Los retos del futuro}

Como lo dice el título de este documento, la historia de la inmunología en Antioquia tiene futuro. Los retos que, a mi parecer, se le presentan a la disciplina y a quienes hoy tienen la responsabilidad de definir su futuro son científicos, pedagógicos y administrativos. Debo insistir en que las siguientes son apreciaciones personales, que después de trabajar tantos años en inmunología y de compartir ideas con colegas internacionales, compañeros y discípulos locales, estimo que 
deben tenerse en cuenta por quienes hoy toman las decisiones para garantizar que la inmunología continúe siendo líder en la investigación biomédica local y que sus aplicaciones tengan un impacto acorde con ese liderazgo. Estas son mis apreciaciones:

En el plano científico se debe mantener, mejorar y ampliar, el nivel y el tipo de investigación que se realiza localmente para adecuarse así a las realidades de la investigación inmunológica en el mundo, la cual, se mueve en direcciones aparentemente opuestas, pero en realidad complementarias: mientras se profundiza en los mecanismos genéticos y moleculares puntuales que controlan la respuesta inmune, se busca superar el enfoque reduccionista, empleado tradicionalmente, con un enfoque sistémico que muestre las interacciones genéticas y moleculares en un contexto más amplio. Un reto es, entonces, acceder a la llamada biología de sistemas (system biology) aplicada a la inmunología, la cual implica, además, asociarse con investigadores formados en matemáticas y ciencias de la computación. Debo reconocer que las recientes colaboraciones del GICIG, en cabeza de Luis Fernando Barrera y Andrés Baena junto con el Centro Nacional de Secuenciación Genómica e investigadores de la Facultad de Ingeniería de la Universidad de Antioquia, apuntan en esa dirección. Otro reto que creo importante es trabajar más intensamente en la que denomino la "época de la cosecha”. Me refiero al auge actual de la inmunoterapia aplicada a condiciones como las enfermedades autoinmunes y, muy especialmente, al cáncer, entre otras, ya que al haberse empleado herramientas derivadas de los largos años de investigación fundamental se ha cambiado radicalmente la atención de los pacientes con estas enfermedades, brindándoles una mejor calidad de vida y unas posibilidades de supervivencia insospechadas hasta hace unos pocos años. Estos desarrollos abren las posibilidades para nuevos descubrimientos y requieren, además, un componente investigativo muy sólido en su aplicación, al buscar biomarcadores inmunes para la selección adecuada de los pacientes y su seguimiento durante el tratamiento inmunoterapéutico. No creo equivocarme si digo que aún no hay una actividad significativa de parte de los grupos de inmunología locales en esta dirección.

Otra área que considero muy promisoria en un país como Colombia es el estudio del sistema inmune en especies diferentes a la de los humanos. Una de las grandes riquezas de nuestro país es su biodiversidad, pero hasta ahora el estudio de esta se ha concentrado en el levantamiento de inventarios en diferentes áreas geográficas, sin que hayamos empezado a estudiar los mecanismos biológicos, incluyendo los inmunológicos, que poseen esa multitud de especies que han demostrado estar capacitadas para colonizar exitosamente tantos nichos ecológicos diferentes.

La enseñanza de la inmunología también enfrenta retos pedagógicos significativos para lograr que la docencia en pregrado y posgrado, tanto de tipo básico como clínico, esté acorde con los desarrollos científicos y las traducciones que de estos suceden en la atención de múltiples estados inmunopatológicos, como lo mencioné anteriormente. En lo que respecta a los pregrados y posgrados clínicos me pregunto, si estamos formando adecuadamente a los médicos y a los especialistas para comprender y utilizar en forma óptima las herramientas diagnósticas y terapéuticas disponibles en esta "época de cosecha”. En los programas de maestría y doctorado creo que continuamos con una estrategia pedagógica muy reduccionista en el planteamiento de los problemas, en el diseño de los experimentos, la selección de procedimientos técnicos, así como en los métodos de análisis que les planteamos a nuestros estudiantes. Me preocupa, igualmente, que los programas de maestría y doctorado básicos hayan dejado de ser atractivos para los médicos y, que los graduados de otros programas de pregrado, una vez que son admitidos en un posgrado biomédico, no adquieran un conocimiento profundo de las patologías sobre las cuales están investigando y que su interés radique en una célula, una molécula o un gen, ionorando los pacientes y las comunidades afectados por tales patologías, quienes han permitido que se obtengan de ellos los especímenes clínicos requeridos para el trabajo de investigación del profesional biomédico. Es decir, se deja de reconocer que es el enfermo mismo quien con su sufrimiento, permite llevar a cabo los estudios investigativos que emprenden los universitarios biomédicos. No se debe olvidar que el paciente merece ser respetado y tenido en cuenta, ya que es la fuente misma del proyecto de investigación en cuestión.

Finalmente, desde el punto de vista administrativo, estimo que es necesario lograr una mayor interacción entre los grupos que trabajan inmunología (además de estos entre sí) con los clínicos que manejan los 
pacientes con patologías mediadas por la respuesta inmune. Es necesario también lograr una mayor incidencia en las políticas de la Universidad y del país en lo referente a la investigación, el manejo de recursos, los programas de posgrado y la selección de estudiantes. Me preocupa que, en general, haya tan poca interacción académica y colaborativa entre los grupos cuando, en muchas ocasiones, parecen competir en vez de colaborar y compartir. Postulo tres posibilidades, igualmente como temas de análisis para quienes hoy son responsables del futuro de nuestra disciplina:

1. Muchos de los colegas internacionales que nos ha visitado han planteado la posibilidad de postuIarnos como centro FOCIS ("Federation of Clinical Immunology Societies"), lo cual les daría una mayor visibilidad a los grupos y a la Universidad de Antioquia, además de que facilitaría el acceso a entrenamientos, becas, asesorías y proyectos de investigación colaborativos internacionales. En América Latina hay varios centros FOCIS y todos ellos reconocen que han obtenido beneficios importantes por haberlo logrado. Un requisito para ello sería una propuesta hecha por varios grupos que demostrará que, efectivamente, se tiene actividad investigativa, docente y clínica en varias áreas de la inmunología

2. La creación de un instituto de inmunología en la Universidad de Antioquia. Contamos para ello con todo lo que los reglamentos universitarios exigen para serlo, solo tendríamos que demostrar la disponibilidad y la aceptación de los diferentes grupos para formar una unidad administrativa que les diera la independencia y la autonomía académicoadministrativa, que no es posible lograr cuando sus profesores hacen parte de múltiples dependencias.

3. La creación de una especialidad (residencia) en inmunología clínica, que incorpore a la de alergología ya existente y prepare clínicos para la aplicación de los grandes logros de la investigación en inmunología básica en el manejo de pacientes con errores innatos de la respuesta inmune, enfermedades infecciosas, autoinmunes, neoplásicas y metabólicas crónicas.

Para finalizar, solo me resta afirmar que estas cinco décadas en las que he trabajado en inmunología en la Universidad de Antioquia, bajo la guía de mis maestros y, luego en compañía de mis colegas y discípulos, le han dado sentido a mi vida personal y profesional. A la Institución y a todos ellos me debo como científico y como persona.

\section{AGRADECIMIENTOS}

A Fabiola Montoya de Restrepo, Marcos Restrepo y William Rojas por haberme concedido gentilmente entrevistas en las cuales recopilé información y materiales muy valiosos para esta reseña. A Diana García de Olarte por sus aportes al documento y la fotografía que gentilmente me proporcionó. A Ángela Restrepo, Sara Claudia París, Luis Fernando Barrera y Gloria M. Vásquez por la lectura crítica y sus aportes a la forma y el contenido del presente documento.

\section{REFERENCIAS BIBLIOGRÁFICAS}

1. Arias M, Rojas M, Zabaleta J, Rodríguez JI, París SC, Barrera LF, et al. Inhibition of virulent Mycobacterium tuberculosis by Bcor and Bcos macrophages correlates with nitric oxide production. J. Infect Dis. 1997;176:1552-8. DOI 10.1086/514154.

2. Pan1 H, Yan B-S, Rojas M, Shebzukhor XV, Zhou H, Kobzik L, et al. Ipr1 gene mediates innate immunity to tuberculosis. Nature. 2005;434(7034):767-72. DOI 10.1038/nature03419.

3. Van Zelm MC, Reisli I, van der Burğ M, Castaño D, van Noesel CJM, van Tol MJD, et al. An antibody deficiency syndrome due to mutations in the CD19 gene. N Eng J Med. 2006;354(18):1901-12. DOI: 10.1056/NEJMoa051568.

4. Arias MA, Jaramillo G, López YP, Mejía N, Mejía C, Pantoja AE, et al. Mycobacterium tuberculosis antigens specifically modulate CCR2 and MCP-1/CCL2 on lymphoid cells from human pulmonary hilar lymph nodes. J Immunol. 2007;179(12):8381-91. DOI 10.4049/jimmunol.179.12.8381.

5. Del Corral H, París SC, Marín ND, Marín DM, López L, Henao HM, et al. IFNg Response to Mycobacterium tuberculosis, risk of infection and disease in household contacts of Tuberculosis patients in Colombia. PLoS ONE 2009;4:e8257-e. DOI:10.1371/journal. pone.0008257. 